\title{
An examination of Pavlovian induction phenomena in differential instrumental conditioning'
}

DALE W. LEONARD, JON WEIMER, AND RICHARD ALBIN, DEPARTMENT OF PSYCHOLOGY, UNIVERSITY OF ROCHESTER, Rochester, N. Y. 14627

Thirty three albino rats learned a successive $B-W$ discrimination in Phase I and were then tested for induction in Phase II. Both positive and negative induction-like effects appeared in Phase II, but no evidence for induction was found during Phase I discrimination training. The results were seen to be more nearly in line with a simple generalization decrement hypothesis rather than the classical Pavlovian induction hypothesis.

Pavlov's (1927) early work on the classically conditioned salivary response in dogs introduced the concept of induction into the psychological literature. In Pavlov's experiments on induction Ss were trained to discriminate between two stimuli, one reinforced (CSt), the other nonreinforced (CS-). Positive induction was demonstrated by increased salivation on those CS+ trials which immediately followed one or more CS- trials. According to Pavlov (1927, p. 189) the negative CS- enhanced the excitability of the cortical area corresponding to the focus of the CS+. Based on this analysis, positive induction might also be indicated by greater resistance to extinction of the conditioned response (CR) to CS+ when extinction trials to CS+ are alternated with presentations of CS- as opposed to extinction obtained by repeated nonreinforcements of $\mathrm{CS}+$ alone. The present investigation presents evidence bearing on both of these methods for demonstrating positive induction.

Negative induction refers to the enhanced negativity of the CSthrough the action of the CSt. This phenomenon was demonstrated in Pavlov's lab by reinforcing CS-following discrimination training. Alternating presentations of CS+ were observed to retard reacquisition of the $\mathrm{CR}$ to the former $\mathrm{CS}$-, whereas reacquisition proceeded quite rapidly with repeated reinforced presentations of CS- alone. In addition, negative induction might also be indicated by decreased response strength on CS- trials which follow one or more CS+ presentations in the latter stages of discrimination training.

A recent experiment (Senf \& Miller, 1967) has shown that the positive induction effect may be present in the instrumental, as well as in the classical conditioning paradigm. After discrimination training in a straight alley (CS+ = flashing lights and white noise; CS- = no lights, no noise) Ss were extinguished with alternating presentations of CS+ and CS- (positive induction group) or by successive presentations of $\mathrm{CS}+$ alone without reinforcement (control). An additional control group ran a neutral ramp on alternate trials. The positive induction group was found to be more resistant to extinction than either of the control groups, which did not differ themselves.

The present investigation attempts to extend the Senf and Miller results by (a) examining negative, as well as positive induction in instrumental learning, and (b) examining the discrimination learning phase itself to determine whether running speed to CS+ is faster following CS- (positive induction), and whether running speed to CS- is slower following CS+ (negative induction).

Subjects. The 33 Ss were experimentally naive, 90-day-old male albino rats purchased from the Holtzman Company, Madison, Wisconsin.

Apparatus. The differential conditioning box consisted of two parallel $36 \mathrm{x}$ $3^{1 / 2} \times 4$ in. alleyways, one painted white, the other black. A single 12 in. gray start box, positioned on rollers, was used with either alleyway. The apparatus was covered with a hinged top of clear Plexiglas. Baffles at the end of both alleys concealed an aluminum goal cup into which Noyes $.037 \mathrm{~g}$ pellets could be placed. A Hunter Klockcounter, controlled by the start door and a photocell 12 in. down the alleys, recorded start times to the nearest $.01 \mathrm{sec}$.

Procedure. Prior to training, Ss received 11 days on a $14 \mathrm{~g}$ per day food deprivation schedule, during which they were individually handled and given a total of 10 rewarded (six pellet) goal placements, five in each alley. Following pretraining, a 10 day period of successive discrimination training was begun. Ss received eight trials per day, four to each of the white (CS+) and black (CS-) alleys according to a prearranged irregular schedule. On CS+ trials Ss were removed as soon as they had consumed the six pellet reward; on CS- trials Ss were detained in the empty goal box for 10-15 sec. Ss were run in squads of four so that intertrial interval (ITI) was determined by the time needed to run the other three $S s$ in a squad, approximately 3-5 min.

Induction Tests. On the day following discrimination training, six Ss were randomly assigned to each of two positive induction groups, and seven $S s$ to each of three negative induction groups. During the tests for induction, each $S$ ran all its trials consecutively, rather than in rotation.

The positive induction experimental group (PI-E) received 24 trials, alternating between the CS+ and CS- alleys, but was nonrewarded in both alleys in Phase II. The control group (PI-C) also received 12 extinction trials in the CS+ alley, alternated with 12 nonrewarded placements in a neutral gray goal box, apart from the alley.

The negative induction experimental group (NI-E) received 24 trials, alternating between the CS+ and CS- alleys, with six pellets of reward given in both alleys. One control group (NI-C) received 12 consecutive rewarded trials in the CS- alley with a $15 \mathrm{sec}$ wait between trials to help equate its ITI to that of Group NI-E. A second control group (NI-P) was given 12 rewarded trials in the CS- alley alternated with 12 rewarded placements in the neutral box during Phase II.

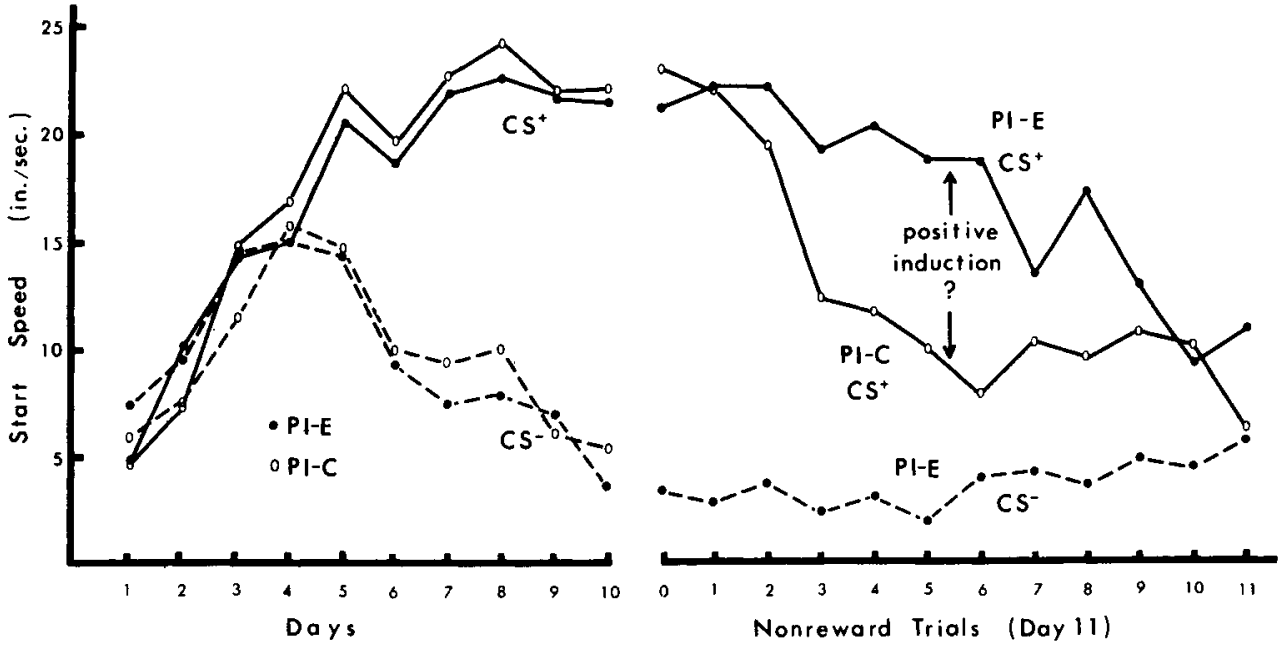

Fig. 1. Speeds in the CS+ and CS- alleys during discrimination training and in the tests for positive induction. 

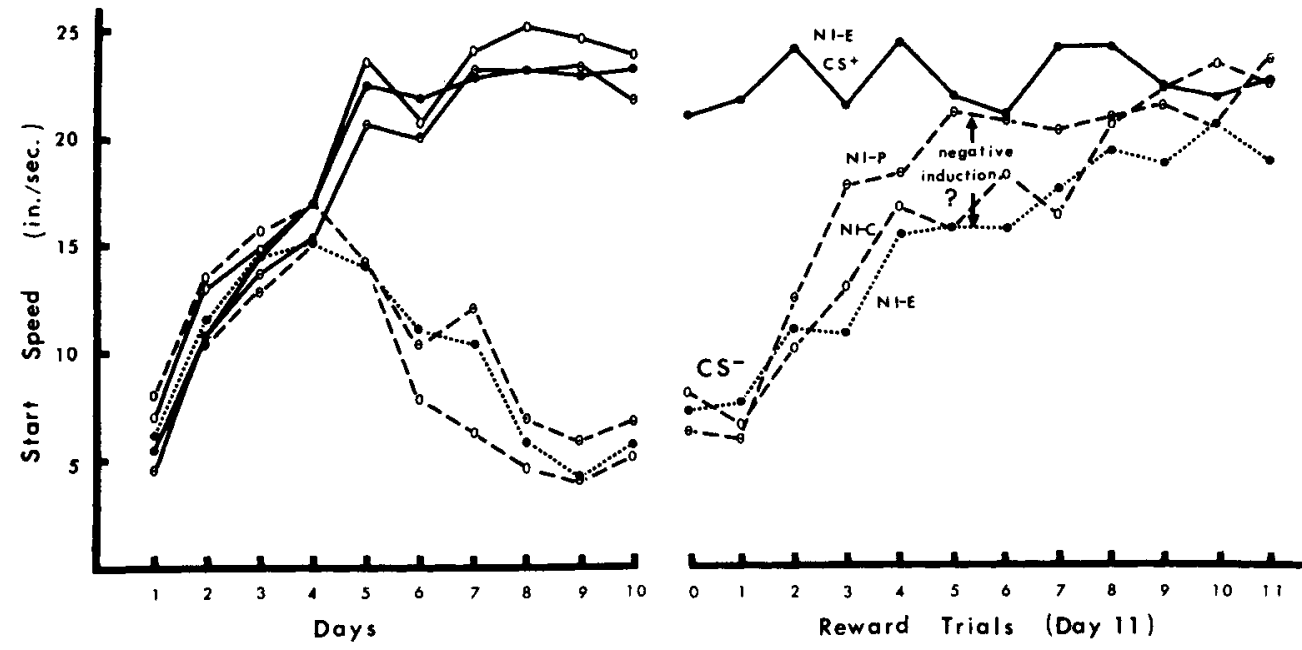

Fig. 2. Speeds in the CS+ and CS- alleys during discrimination training and in the tests for negative induction.

\section{Results}

The start times were first converted to speeds (in/sec) for purpose of analysis. The last two days of discrimination training, Days 9-10, were used to determine whether or not induction effects were present in Phase I. ${ }^{2}$ Data from all 33 Ss indicated that Ss ran slightly faster ( $23.7 \mathrm{vs} 22.1 \mathrm{in} / \mathrm{sec})$ on CS+ trials which were preceeded by CS+ rather than CS-. This difference was reliable $(t=$ 3.56 , $\mathrm{df}=32, \mathrm{p}<.01)$ and contrary to that expected from the theory of positive induction. Ss also ran somewhat slower (4.5 vs $6.2 \mathrm{in} / \mathrm{sec}$ ) on CS- trials preceeded by CS+ rather than CS-, a result in line with the negative induction effect, but this difference was not significant $(\mathrm{t}=1.89, \mathrm{df}=32, .05<\mathrm{p}<.10)$.

Figure 1 presents the Phase I and Phase II performances of the positive induction groups, PI-E and PI-C. All Ss learned to discriminate the CSt and CS- alleys in Phase I. With all trials nonreinforced in Phase II, Fig. 1 shows that Group PI-E exhibited greater resistance to extinction in the former CS+ alley compared to Group PI-C, despite the fact that the latter group experienced only half as many nonrewarded trials in Phase II. This difference, indicating a strong positive induction effect, was significant by the Mann-Whitney Test $\left(U=5, n_{1}=n_{2}=6, p=.021\right)$. The speeds of Group PI-E on the 12 trials in the CS- alley are also plotted in Fig. 1.

Figure 2 presents the data from the three groups tested for negative induction effects. Again all subgroups discriminated equally well in Phase I. In Phase II, with CS- now rewarded, a significant difference indicating a negative induction effect, was found between Group NI-E and the NI-P control group $(\mathrm{U}=10$, $n_{1}=n_{2}=7, p=.036$ ). However, no difference was obtained between NI-E and the other control, NI-C $\left(U=23, n_{1}=n_{2}=7, p\right.$ $=.451)$. Therefore, although Group NI-P is in many respects a more appropriate control, since total rewards are equated, the evidence for negative induction found here should nonetheless be interpreted with caution.

Discussion

Judging from the present results and those of Senf \& Miller (1967), it appears to be relatively easy to obtain positive induction-like phenomena by comparing the extinction of an instrumental response to a former CS+ with or without the presence of CS-. There was also a suggestion in the present investigation that negative induction-like effects may be produced in a corresponding fashion.

However, the absence of any significant induction ef fects during original discrimination training (in fact, a significant inverse positive induction effect was found) casts doubt on the induction concept as an explanatory mechanism for these data. It should be pointed out that the induction-like effects of Phase II can be most simply attributed to greater generalization decrement in the control groups, who necessarily undergo the greatest stimulus change during the induction tests. ${ }^{3}$ Frey \& Ross (1967), in their study of induction involving the classically conditioned nictitating membrane response in rabbits, reached a similar conclusion. Here again induction-like effects appeared under conditions involving greater stimulus change for the controls, but no evidence for induction was found during original discrimination training.

\section{REFERENCES}

FREY, P. W., \& ROSS, L. E. Differential conditioning of the rabbit's eyelid response with an examination of Pavlov's induction hypothesis. J. comp. physiol. Psychol, 1967, 64, 277-283.

PAVLOV, I. P. Conditioned reflexes. (Trans. by G. V. Anrep) London: Oxford University Press, 1927.

SENF, G. M., \& MILLER, N. E. Evidence for positive induction in discrimination learning. J. comp. physiol. Psychol, 1967, 64, 121-127. NOTES

1. The authors are indebted to R. V. Krane and J. R. Ison for their advice in the design of this experiment, and for the use of their equipment which was provided by NSF Research Grant GB 4373.

2. The reward sequence on Day 9 was $++\ldots+++$. Day 10 employed the opposite sequence $-\cdot+++-+$. .

3. Senf and Miller tried to control for stimulus change in one of their experiments by using a control group of six Ss who received a long run of CSt stimuli prior to induction tests. These experimenters still found induction effects upon subsequent testing. Whether or not this group represents an adequate control for stimulus change remains to be seen. 